\title{
"Development of Basic Skills in Swimming Sports Training At Grassroot Level through Distance Education Method: A Case Study on School Going Children".
}

\author{
Dr. Atmaram Vaman Thoke \\ Director of Physical Education, Department of Physical Education \\ SVKT Arts, Science and Commerce College, Deolali Camp, Nashik- 4224001 (Maharashtra State) India
}

\begin{abstract}
The management and planning of sports training at grass root level is an executive function, which is concerned with carrying out of the broad policies laid down for the training of upcoming youngsters. Such training is an urgent need, which involves working on different training models and methods and, at the same time, testing their efficiency and communicating it to concerned sport persons. In today's competitive world, it is necessary that a continuous search be carried out to tap hidden talent at early age. In this regard, appropriate methods of training must be used to develop budding sportsmen. In the last decade of this century, we have witnessed the significant impact of communication media in almost all branches of human life and sports are no exception. In the good old days, basic skills of any game and swimming, in particular, was a matter concerning only swimmers and their respective coaches? Frankly speaking, it was all subjective, although some exceptions may be cited. At present, we have audio-visual aids that attract beginners, keen in the developing the basic skills of the sport.
\end{abstract}

We report below the detail of an experiment conducted to assess the efficiency of (a) traditional method (T1), (b) Audio-visual methods (T2) and (c) combination of traditional and audio-visual methods (T3), on imparting and developing the basic skills in the sport of swimming among the school going children. In other words, the experiment was at aimed at assessing the development of swimming skills of school going children through Distance Education method.

The experiment was designed, planned and performed over a span of six weeks in year 1999, in the small town Nashik of Maharashtra State (India). The experiment was performed on 90 school going children. These students were randomly allocated to three different groups G1, G2 and G3 corresponding to T1, T2 and T3 treatments.

Our analysis confirms that we must now use communication techniques to impart basic instructions in the sports of swimming. This does not mean that the traditional method, which heavily depends upon expertise of individual coaches, can be eliminated. In fact a proper combination of treatment 1, i.e. traditional method and treatment 2, i.e. new audio-visual communication media can be blended together as we demonstrated in the formulation of treatment 3. Thus, the effectiveness of communication media will be further improved, if it is supported by the experience of traditional methods.

In other words, our experiment underlines the importance of combined effect of tradition and new communication media in imparting the basic skills to budding sportsmen more effectively by Distance Education method.

\section{Introduction:}

India is a large country with vast potential to accept the challenges in diverse fields of life. However for reasons, known and unknown, the nation as a whole still lacks a sports culture. It is necessary that we make best use of a giant network of universities, colleges and schools and of students placed in these institutes running through across the country to create and impart a sports culture It is many times suggested that we should tap the children at very young age and impart them a special training in the interest of the children them selves and the nation. Training plans have become an integral part of a sport at any level and particularly so when it is a competitive game at State, National or International level. A change either small or radical can be implemented through training plans.

But with the era of communication we are forced to accept the new communication techniques in every field and particularly so in the field of sport. Training programmes and plans need to be monitored, tested objectively and, in turn, the entire methodology must be reexamined using modern tools. Quantitative studies must be initiated wherever possible and these reports must be brought to the notice of concerned players, teachers, sport directors, and people at large. The Aim of sports training can be achieved only if scientific designs already tested are used in a systematic manner. 


\section{Key of works:}

To find out the new methodology in swimming for the development of human being, to remove the fear of water, to update the knowledge \&avoid the threats. To give scope to the inherent qualities\& to increase the status of swimming. To increase the curiosity \& interest among the people by showing video clips. Benefits of practical knowledge. Production of Audio-visual aids. Competitive preparation, physical fitness, happy human life. Self study physical fitness. Make people sports aware of education \& increase in the quality. Increase in scientific knowledge.

Aim \& Object of the study:

1) Development of the basic skills in swimming.

2) Awareness \& development of the knowledge related to swimming.

3) TO removing the fear of water. about swimming skills though communication. communication skills a video clips. methodology \& compare them. methodologies in swimming.

4) To give information

5) To find out the change though

6) To do comparative study of training 7) To find effectiveness of training

\section{Methods \& Material:}

The experiment conducted assessed the efficiency of (a) traditional method (T1), (b) Audio-visual method (T2) and (c) the combination of traditional and audio-visual methods (T3), on imparting and developing the basic skills of the sports of swimming among school going children through the distance education method. The experiment was designed, planned and performed over a span of six weeks in Nashik in the state of Maharashtra (India) in the year 1999. The target group was 90 school going children between the ages ranging form 6to 11 years ages. These students were randomly allocated to three different groups G1, G2 and G3 corresponding to T1, T2 and T3 treatments.

T1 can be called the traditional method. It is a subjective method of imparting basic instructions to beginners, which forms the potential population for a special training in future. The method relies heavily on individual coaches and their expertise. In order to have uniformity amongst the individual coaches a short-term workshop was conducted the authors which the necessary information and details were spelled.

Treatment T2 consisted of imparting instructions to subjects only through audio-visual media. A set of audiovisual cassettes was prepared by the authors and was made available to group 2 (G2). The study material was thus available to the subjects and they could use the same as per their individual needs. Treatment 3 , labeled as T3, is a combination of T1 and T2. Five experts on a 10-point scale judged the performance and progress of the students.

The scores were awarded for the

(a) Fear Removing Test (FR),

(b) Submerging Test (SM),

(c) Floating Test (FL),

(d) Gliding Test (GL) and

(e) Full Stroke Forward Movement Test (FSFM).

The FR test was performed before the beginning of the experiment and also at the end of $2^{\text {nd }}, 4^{\text {th }}, 6^{\text {th }}$ week. SM and FL test was performed at the end of $2^{\text {nd }}, 4^{\text {th }}, 6^{\text {th }}$ week. The GL and FSFM test were performed in $4^{\text {th }}$ and $6^{\text {th }}$ week only. These data based on scores were analyzed in order to test the efficiency of the treatments.

\section{Material Observation, Analysis \& Discussion:}

The comparison and effect of treatments can be evaluated in a scientific manner using the principles of Statistics when the homogeneity of the subject is assured. Table 1 below gives the values of scores (mean \pm s.d) of the three groups to which these treatments were respectively applied. Table 1 clearly demonstrates that each of the treatments is meaningful and does build a confidence among the beginners. Moreover the progress of all the groups was satisfactory however the improvements in the subject differ.

Table: 1

\begin{tabular}{|c|c|c|c|c|c|}
\hline Treatment & Test & Before the trial & Week 2 & Week 4 & Week 6 \\
\hline T1 & $\begin{array}{l}\text { FR (Points) } \\
\text { SM (sec) } \\
\text { FL (meter) } \\
\text { GL (meter) } \\
\text { FSFM (point) }\end{array}$ & $6.70 \pm 1.2$ & $\begin{array}{l}4.50 \pm 0.73 \\
1.4 \\
3.5\end{array}$ & $\begin{array}{l}3.70 \pm 0.75 \\
4.3 \\
4.3 \\
4.6 \\
3.5 \\
\end{array}$ & $\begin{array}{l}0.53 \pm 0.82 \\
7.5 \\
7.5 \\
7.4 \\
5.2 \\
\end{array}$ \\
\hline $\mathbf{T} 2$ & $\begin{array}{l}\text { FR } \\
\text { SM } \\
\text { FL } \\
\text { GL } \\
\text { FSFM } \\
\end{array}$ & $6.70 \pm 1.21$ & $\begin{array}{l}4.43 \pm 0.77 \\
2.5 \\
3.5\end{array}$ & $\begin{array}{l}2.63 \pm 1.30 \\
5.5 \\
5.5 \\
4.5 \\
3.9 \\
\end{array}$ & $\begin{array}{l}0.28 \pm 0.48 \\
7.8 \\
7.8 \\
8.0 \\
5.7\end{array}$ \\
\hline T3 & FR & $6.67 \pm 1.15$ & $3.83 \pm 0.75$ & $4.50 \pm 0.61$ & $0.17 \pm 0.38$ \\
\hline
\end{tabular}




\begin{tabular}{|l|l|l|l|l|l|}
\hline & SM & & 1.5 & 6.6 & \\
& FL & & $\mathbf{7 . 9}$ \\
& GL & & 6.7 & 8.0 \\
& FSFM & & 4.4 & 7.8 \\
\hline
\end{tabular}

Scores of Test Based on 30 subjects in each group

Amongst the three groups the group receiving T3 treatment showed the most progress, where as group 2 and group 1 showed almost the same level of improvement or nearby each other. It simply means that the present day communication techniques combined with traditional methods should be employed to accelerate the learning capacity of individual swimmers.

\section{Conclusion and Discussion:}

It has been suggested many times that a SMART objective plays a role. It stands for Specific, Measurable, Achievable, Realistic and Time bound activity. All these systems usually recommend various approaches, which are claimed to be effective. These claims need to be tested in a scientific manner. Our analysis underlines the importance of combined effect of traditional approach and the role of modern communication techniques. If these two are combined skillfully then satisfactory results can be expected to all. Undoubtedly, these techniques have proved beneficial to newcomers but to know the exact role of similar techniques, scientific experiments need to be designed.

The analysis of a well-planned experiment only can bring out the contribution of modern technological methods in imparting training to children through competitive sports. The current approaches also need to be examined statistically by developing accurate statistical models. The distance education can play a meaningful role particularly in the remote areas wherein we expect an untapped resource of talent in sport. To conclude, our experiment shows ways to propose and evaluate different methodologies of imparting training in swimming. These experiments need to be replicated in different sports under the careful guidance of experts in the field of physical education and educational statistics.

\section{References:}

[1]. Dr. A.V.Thoke ("Primary Skill Development and Evaluation of Swimming in Physical Education through Video Cassette", Ph.D. thesis submitted to YCMOU, Nashik422005, Year 1999.

[2]. Dr. A.V. Thoke and R. Pokharapurkar " Development of Basic Skills in Swimming Through Distance Education Method" paper read in the conference on "Planning and Management of Open and De System: Visions, Paths, Experience and Development" held at Nashik in the year 2000 .

[3]. Dr. A.V. Thoke."AADHUNIK VA SHASTRIYA JALTARAN PRASHIKSHAN”, (Marathi) Priyanka Prakashan, Nashik-422005, Year 2001. 